\title{
New directions in implicit motive research: a special issue
}

\author{
Hugo M. Kehr • Todd M. Thrash • Rex A. Wright
}

Published online: 27 August 2011

(C) Springer Science+Business Media, LLC 2011

Implicit motives are at the heart of motivation psychology. They have roots in the earliest psychological theorizing (e.g., Freud 1966) and have attracted special attention since the late 1980s when scholars such as McClelland (1987) began systematically distinguishing implicit and explicit (or self-attributed) motivation processes. Theory has advanced and led to empirical analyses of mechanisms that link implicit motives with affective, autonomic and cortical responses as well as with self-regulation and volitional behavior. Investigators have proffered new motive taxonomies that distinguish types of implicit motives and begun to study moderators and consequences of implicit-explicit motive discrepancies. Practitioners have become interested in the management of implicit motives and implicit motive influence on leadership.

Our purpose in organizing this Special Issue was to provide in one place a sampling of cutting edge implicit motive reasoning and research and, in doing so, raise further the professional profile of this diverse and expanding area of interest. We aimed to sample broadly so as to provide a reasonable flavor of current trends. At the same time, we wanted to maintain a focus on theory, showing a preference for papers that developed new conceptual analyses or evaluated existing ones. Much to our satisfaction, we had no difficulty meeting our aims.

H. M. Kehr (

Technical University of Munich, Munich, Germany

e-mail:kehr@wi.tum.de

T. M. Thrash

College of William and Mary, Williamsburg, VA, USA

R. A. Wright

University of Alabama at Birmingham, Birmingham, AL, USA
Papers that we accepted were varied in numerous respects, but were sufficiently thematic to allow us to structure the issue into three sections. Section 1 includes three articles concerned with implicit motive assessment. It begins with an article by Bernecker and Job that presents findings from a study designed to evaluate the validity of an online version of the Picture Story Exercise-a projective test through which implicit motives commonly are assessed. As expected, data collected online were highly correspondent to data collected in a conventional laboratory context, suggesting that the online version could be utilized when conventional laboratory data collection is not possible or practical.

The section continues with an article by Baumann and Scheffer that discusses work that used a modified version of the Operant Motive Test (Kuhl and Scheffer, 1999) to assess the implicit flow motive underlying the phenomenological flow experience. The authors present four studies that support the notion that the achievement flow motive can be conceptualized as the intrinsic component of the achievement motive.

Concluding this section, an article by Ostafin and Brooks introduces a novel version of the Implicit Association Test, an assessment instrument that is based on reaction times, to assess automatic alcohol motivation. Participants categorized stimuli from two target categories (e.g., pictures of beer and water) and two attribute categories (e.g., approach and avoidance-related words). The authors found evidence for the long-held assumption that negative affect can increase the strength of automatic motivational processes related to alcohol.

Section 2 includes three articles concerned with energy efficiency and motive congruence. Morsella, Feinberg, Cigarchi, Newton and Williams note that organisms must conserve energy to function effectively. Based on this, they 
suggest that organisms might have evolved a mechanism that leads them to avoid reflexively stimuli associated with high effort expenditure. The investigators present supportive findings from two experiments, discussing implications of the findings and their fit with other relevant theories. Schattke, Koestner, and Kehr present the first published study of childhood antecedents of motive incongruence. Building upon McClelland and Pilon's (1983) study of archival data (Sears et al. 1957), they find that several maternal socialization practices when a child is 5 -year-old predict motive incongruence 26 years later. Kazén and Kuhl examine the effect of motive incongruence on well-being among managers. This article is particularly innovative in that the authors analyze motive congruence using polynomial regression with response surface methods, a 3-dimensional modeling technique that permits separate testing of two forms of incongruence that are confounded when more traditional techniques are used.

The third section of the Special Issue includes two articles that address cross-country concerns. $\mathrm{Ng}$, Winter, and Cardona analyze power motivation from a cross-cultural perspective and present findings from a relevant experiment. Results of the experiment comport with their suggestion that having decision-making control over resources increases levels of power motivation among Americans, but not Chinese. Szeto, Sorrentino, Yasunaga, Kouhara, and Lin expand the theory of uncertainty orientation (Sorrentino and Roney 2000) to embrace the implicit achievement motive. They present findings from a crosscultural study in Canadian and Japanese education settings which indicate a triple interaction effect of students' uncertainty orientation, their culture's dominant style of uncertainty regulation (for Japan: maintaining certainty; for Canada: resolving uncertainty) and the students' achievement motive with respect to grades.
Together, the articles in this special issue illustrate that the implicit motive literature is flourishing. Methodological innovations include updates to the venerable Picture Story Exercise and its progeny, application of objective reactiontime methods, and the introduction of sophisticated modeling techniques that keep pace with recent theoretical developments. Implicit motives are shown to have broad theoretical relevance, serving as foundational constructs in research on self-regulation, flow, well-being, development, decision-making, and uncertainty. Finally, implicit motive research is addressing an important contextual factor, culture, which will become increasingly important as the world moves toward globalization and democratization. As long as the implicit motive approach continues to wed the best ideas from its intellectual history with cutting-edge methodological and theoretical innovations, it will continue to thrive.

\section{References}

Freud, S. (1966). Introductory lectures on psychoanalysis (J. Strachey, Trans.). New York: Norton.

Kuhl, J., \& Scheffer, D. (1999). Der operante multi-motiv-test (OMT): Manual [Scoring manual for the operant multi-motive test (OMT)]. Osnabrück, Germany: University of Osnabrück.

McClelland, D. C. (1987). Biological aspects of human motivation. In F. Halisch \& J. Kuhl (Eds.), Motivation, intention, and volition (pp. 11-19). Berlin: Springer.

McClelland, D. C., \& Pilon, D. A. (1983). Sources of adult motives in patterns of parent behavior in early childhood. Journal of Personality and Social Psychology, 44, 564-574.

Sears, R. R., Maccoby, E. E., \& Levin, H. (1957). Patterns of child rearing. Evanston, IL: Row Peterson.

Sorrentino, R. M., \& Roney, C. R. J. (2000). The uncertain mind: Individual differences in facing the unknown. Philadelphia, PA: Psychology Press. 